J. Lake Sci.(湖泊科学), 2019, 31(1): 293-304

DOI 10. 18307/2019. 0127

(c) 2019 by Journal of Lake Sciences

\title{
1860 年以来博斯腾湖碳沉积过程演变
}

于志同 $^{1,2,3}$, 李广宇 ${ }^{4}$, 张恩楼 ${ }^{5}$, 刘兴起 ${ }^{6}$

(1: 中国空间技术研究院钱学森空间技术实验室,北京 100094)

(2: 北京师范大学全球变化与地球系统科学研究院, 北京 100875)

(3: 中国科学院新疆生态与地理研究所荒漠与绿洲国家重点实验室, 乌鲁木齐 830011)

( 4 : 环境保护部南京环境科学研究所,南京 210042)

(5: 中国科学院南京地理与湖泊研究所湖泊与环境国家重点实验室, 南京 210008)

(6: 首都师范大学资源环境与旅游学院, 北京 100048)

摘 要: 选取博斯腾湖 3 个不同点位岩芯, 在 ${ }^{210} \mathrm{~Pb}$ 和 ${ }^{137} \mathrm{Cs}$ 年代序列基础上,利用沉积物中的各理化指标, 分析了该湖碳 沉积的时空变化特征,结合各指标的相关性、沉积速率、C/N、同位素特征等,探讨了该湖 1860 年以来碳沉积环境的变化 过程, 为干旱区湖泊碳埋藏研究提供了依据. 结果表明: 1860-1910 年, 沉积速率相对较小, 受人类活动影响较小, 磁化 率、中值粒径、总无机碳 (TIC) 较为稳定, 总有机碳 ( TOC) 含量相对较低, 此时该湖有较多陆源有机质的输人; $1910-1950$ 年, 湖泊西部浅水域沉积速率明显高于东部深水区, 西北湖区水域有大量外源物质的输人, 而湖泊初级生产力较低, 内源 贡献相对较小; 1950-1980 年, 全湖 TOC、TIC 含量均呈现升高的趋势, 尤其是西北近黄水沟水域升高最快, 湖泊内源贡献 在增加, 陆源组分的输人相对前一阶段要少; 1980-2002 年, 沉积速率快速升高, 尤其湖泊东部水域最为明显, TOC 含量 均呈现升高的趋势, 湖泊西部水域初级生产力要高于东部深水区, 湖面蒸发较强, 气候比较温暖; 2002 年以来, 全湖沉积 速率相对较高, 外源有机质贡献较小. 过去 150 年博斯腾湖沉积物碳累积速率整体上呈现出升高的趋势, 尤其是近 50 年 来快速升高,东部湖区碳累积速率比西部湖区高.

关键词: 博斯腾湖;有机碳;无机碳;沉积速率; 碳沉积过程

\section{Process variations of sedimentary carbon accumulation in Lake Bosten since 1860}

\author{
YU Zhitong ${ }^{1,2,3}$, LI Guangyu ${ }^{4}$, ZHANG Enlou ${ }^{5} \&$ LIU Xingqi ${ }^{6}$ \\ (1: Qian Xuesen Laboratory of Space Technology, China Academy of Space Technology, Beijing 100094, P.R.China) \\ (2: College of Global Change and Earth System Science, Beijing Normal University, Beijing 100875, P.R.China) \\ (3: State Key Laboratory of Desert and Oasis Ecology, Xinjiang Institute of Ecology and Geography, Chinese Academy of Sci- \\ ences, Urumqi 830011, P.R.China) \\ (4: Nanjing Institute of Environmental Sciences of the Ministry of Environmental Protection, Nanjing 210042, P.R.China) \\ (5: State Key Laboratory of Lake Science and Environment, Nanjing Institute of Geography and Limnology, Chinese Academy \\ of Sciences, Nanjing 210008, P.R.China) \\ (6: College of Resource Environment and Tourism, Capital Normal University, Beijing 100048, P.R.China)
}

\begin{abstract}
Despite the small coverage on the earth, lakes play an important role in the terrestrial carbon cycle. There are a large number of lakes in Xinjiang, Northwest China, where remarkable climate changes and human activity have been undergone over the past decades. Lake Bosten, the largest fresh water inland lake in Northwest China, was selected as the study site. We designed to investigate the temporal variability of carbon burial over the past 150 years, by examining the chronology and multi-proxies (i.e. magnetic susceptibility, grain size, organic carbon, inorganic carbon and stable carbon isotopes) of lacustrine sediments in Lake Bosten. The process variations of sedimentary carbon accumulation in Lake Bosten were divided into five stages since 1860. Before
\end{abstract}

* 国家自然科学基金项目(41601107)、中国博士后科学基金项目(2018T110067,2016M600059)、国家科技基础性工 作专项( 2014FY110400) 和学科建设经费项目 (312231103) 联合资助. 2018-03-22 收稿; 2018-06-15 收修改稿. 于志同(1985 ), 男,博士;E-mail : yuzhitong@ escience.cn. 
1910s, environmental changes were mainly affected by the natural factors, with relatively lower sediment accumulation rates and total organic carbon (TOC), and a stable total inorganic carbon (TIC) in the eastern lake area but a downward trend in the northwest part. During 1910s-1950s, the sediment accumulation rates in the western lake was much higher than that in the eastern deep area, characterized with low lake primary productivity and allochthonous dominated organic carbon ( OC). During 1950s-1980s, a rapid increase trend was found for both TOC and TIC, especially in the northwest lake section near the Huangshui River, while OC from allochthonous showed a decrease. The sediment accumulation rate increased significantly in all the three cores in the 1980s$2002 \mathrm{~s}$, a similar trend shown in TOC under a warm climate. After 2002s, the sediment accumulation rate was relatively high, but the contribution from allochthonous sources was small. During the last 150 years, the carbon accumulation rates presented an increasing trend, especially since 1960 , with higher values in the eastern lake area than in the western section.

Keywords: Lake Bosten; organic carbon; inorganic carbon; sediment accumulation rate; carbon deposition process

了解湖泊在自然和人类活动干扰下的动态, 确定湖泊长期的演变过程及规律, 对于科学地管理和保护 湖泊生态环境尤为重要. 而对于大多数湖泊而言, 长时间、连续的水文、气象等监测资料是缺乏的, 给长时间 序列的研究带来一定的困难, 为此需要间接指标来弥补实测数据的空白. 湖泊沉积物具有连续、分辨率高、 时间尺度长、对环境变化反应灵敏等特征, 在全球变化研究中, 尤其是百年尺度的高分率环境重建中有着重 要的作用 ${ }^{[1]}$. 其中, 有机碳、无机碳含量和分布以及碳沉积过程的研究, 是评价湖泊碳源/汇功能的关键.

湖泊沉积物记录了丰富的湖泊及流域内的环境信息, 因不同点位具有不同的沉积环境, 如水动力条件、 水质、物质来源、氧化一还原状况等, 造成各生物地球化学指标(含水量、干容重、粒度、磁化率、有机碳及其稳 定同位素、总氮、无机碳及其稳定同位素等) 的多解性及差异性 ${ }^{[2]}$. 一些研究指出湖泊沉积物中有机碳和无 机碳呈负相关关系, 有机碳能促进无机碳的溶解, 而在一些地区也发现两者呈正相关关系, 即有机碳与无机 碳同步消长 ${ }^{[3-4]}$. 有机碳和总氮一般用来反演湖泊初级生产力的大小, 而碳氮比 $(\mathrm{C} / \mathrm{N})$ 可以大致判断沉积物 中有机质的来源; 同时, 上述指标还常常受到生物扰动、风浪、湖流、有机物再悬浮、矿化等作用影响, 使得 $\mathrm{C} / \mathrm{N}$ 、有机碳、总氮等理化指标之间关系变得更为模糊、复杂, 因地而异 ${ }^{[5-6]}$. 另外, 有机碳同位素可以定量区 分有机质的来源, 受沉积后埋藏环境的影响较小, 比 $\mathrm{C} / \mathrm{N}$ 的经验判断更为准确. 沉积物的粒度参数, 可以记 录沉积物物质搬运、侵蚀、沉积等过程, 而且不同组分的粒径对有机质的吸附能力不同,一般认为较细颗粒 容易吸附并保存有机碳 ${ }^{[2]}$. 对海洋沉积物、三角洲沉积物的研究也证实有机碳与黏土组分呈显著正相关, 而 与粗颗粒呈负相关, 说明细颗粒沉积物中容易保存有机质 ${ }^{[7]}$.

国内对西北内陆干旱区湖泊的碳循环过程研究相对较少, 大部分是利用沉积物中有机碳、无机碳进行 长时间尺度的古气候环境反演与重建 ${ }^{[8-13]}$, 仅蓝江湖 ${ }^{[14]}$ 通过搜集已发表的文献资料初步估算了我国西北干 旱一半干旱区湖泊近 200 年以来的碳埋藏量, 对干旱区湖泊碳库及其动态变化还缺乏认识. 目前, 围绕博斯 腾湖已开展了有机碳空间分布及来源 ${ }^{[15-16]}$ 、无机碳空间分异规律 ${ }^{[17]}$ 、水体各形态碳的时空变化 ${ }^{[18]}$ 等研究, 多针对表层水体及沉积物的碳空间差异问题, 而对长时间尺度的沉积物碳沉积变化过程还不是很清楚. 初 步研究发现 ${ }^{[19-20]}$, 博斯腾湖 1950 年以来沉积物碳埋藏与流域温度和人类活动 (土地利用变化和营养盐的输 人) 有关. 考虑到博斯腾湖水域面积广大、湖区内部差异性等因素, 本文在大湖区内西南、西北和东部水域选 取了 3 个柱状钻孔岩芯进行常规指标分析 (磁化率、粒度、有机碳、无机碳及其稳定同位素等), 基于多指标 分析其环境指示意义,对博斯腾湖碳沉积环境的时空变化进行综合探讨.

\section{1 研究区概况}

博斯腾湖 $\left(41^{\circ} 32^{\prime} \sim 42^{\circ} 14^{\prime} \mathrm{N}, 86^{\circ} 19^{\prime} \sim 87^{\circ} 26^{\prime} \mathrm{E}\right.$ ) 位于新疆天山南麓, 地处巴音郭楞蒙古自治州博湖县 (图 1 ), 是焉耆盆地 (包括焉耆、和静、和硕、博湖四县的平原部分和库尔勒市的塔什店区及兵团农二师 $21 \sim 27$ 等 8 个团的农场) 的最低点. 湖面海拔 $1048 \mathrm{~m}$, 流域面积约为 $55600 \mathrm{~km}^{2}$, 湖区东西长 $55 \mathrm{~km}$, 南北宽 $25 \mathrm{~km}$, 总面积 $1646 \mathrm{~km}^{2}$ ( 2002 年水位最高时数据) ${ }^{[21]}$. 湖区地处深居欧亚大陆中心, 属温带大陆性干旱气候, 生态 环境极为脆弱. 开都河是其唯一常年有水人湖的河流,约占人湖径流量的 $95 \%$; 同时还有黄水沟、清水河、乌 拉斯台河、曲惠河和乌什塔拉河等季节性河流的补给 ${ }^{[22]}$. 博斯腾湖是一个吞吐型湖泊, 在自然情况下, 湖水 经孔雀河从博湖西南角流出, 后经铁门关折而向东注人罗布泊. 自 1950s 以来, 在人类活动和气候变化的双 
重影响下, 湖泊水位多次急剧变化, 波动幅度在 $1044 \sim 1049 \mathrm{~m}$ 之间 ${ }^{[23-25]}$. 湖水交换能力较低, 总碱度达 $193.39 \mathrm{mg} / \mathrm{L}$, 呈弱碱性、高硬度 ${ }^{[21]}$.

\section{2 材料与方法}

\section{1 样品采集}

2012 年 8 月,利用重力采样器在博斯腾湖大湖区采集了表层沉积物样品,在 $\mathrm{B} 3\left(41.93^{\circ} \mathrm{N}, 86.90^{\circ} \mathrm{E}\right) 、 \mathrm{~B} 5$ $\left(41.96^{\circ} \mathrm{N}, 87.21^{\circ} \mathrm{E}\right) 、 \mathrm{~B} 10\left(42.00^{\circ} \mathrm{N}, 86.98^{\circ} \mathrm{E}\right)$ 点位采集到 3 根平行短柱样品, 岩芯长度分别为 $23.5 、 21.5$ 和 $28.5 \mathrm{~cm}$ (图 1, BT04C 引自文献 [9]; BST13\&16 引自文献 [26]). 采样过程中,沉积物一水界面清澈透明,未 受扰动. 短柱子样品室内以 $0.5 \mathrm{~cm}$ 或者 $1 \mathrm{~cm}$ 间隔分样, 用于 ${ }^{137} \mathrm{Cs} \&^{210} \mathrm{~Pb}$ 测年及各理化指标分析.

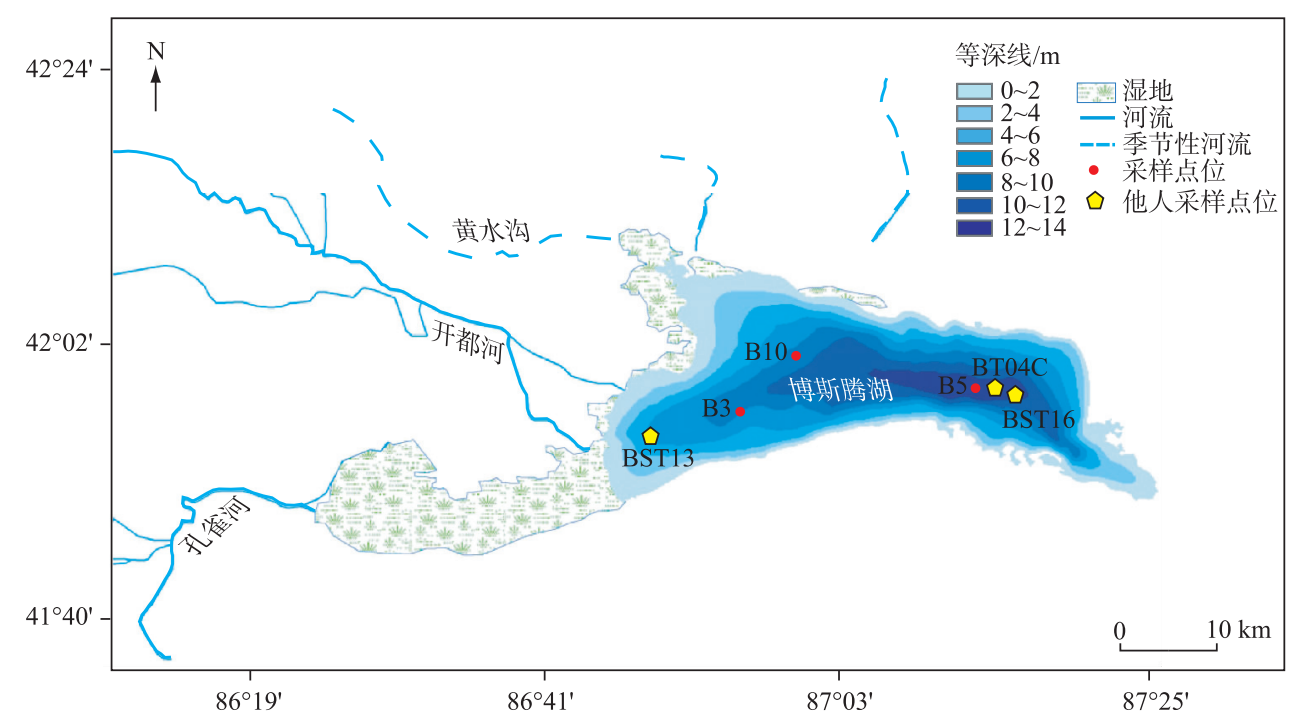

图 1 博斯腾湖湖水等深线及短柱岩芯点位分布

Fig. 1 Distribution of isobaths and the drilling sites in the Lake Bosten

\section{$2.2{ }^{210} \mathrm{~Pb} 、{ }^{137} \mathrm{Cs}$ 样品测试}

实验室内, 将短柱样品进行冷冻干燥处理, 选取适量样品 $(2 \sim 5 \mathrm{~g})$ 研磨至 100 目左右, ${ }^{210} \mathrm{~Pb}$ 和 ${ }^{137} \mathrm{Cs}$ 测 试采用 $\gamma$ 仪分析法, 即样品中 ${ }^{210} \mathrm{~Pb} 、{ }^{226} \mathrm{Ra}$ 以及 ${ }^{137} \mathrm{Cs}$ 活度采用美国 EG \& GOrtec 公司生产的高纯锗井型探测 器( HPGe GWL-120-15) 与 Ortec 919 四型谱控制器和 IBM 微机构成的 $16 \mathrm{~K}$ 多道分析器测定. ${ }^{137} \mathrm{Cs}$ 和 ${ }^{226} \mathrm{Ra}$ 标准样品由中国原子能科学研究院提供, ${ }^{210} \mathrm{~Pb}$ 标准样品由英国利物浦大学做比对标准, 测试误差小于 $10 \%$. 样品年代测定在中国科学院南京地理与湖泊研究所湖泊与环境国家重点实验室进行.

\section{3 理化指标分析}

2.3.1 含水量及干容重分析 含水量 $(W, \%)$ 是指沉积物中所含水分的数量, 是湖泊沉积学中的一个关键性 参数. 通常采用样品中水的质量与湿样质量 $\left(m_{\text {湿 }}\right)$ 之比, 计算公式为:

$$
W=\left(m_{\text {湿 }}-m_{\text {干 }}\right) / m_{\text {湿 }} \times 100 \%
$$

干容重 $\left(D b d, \mathrm{~g} / \mathrm{cm}^{3}\right)$ 是指沉积物在自然状态下单位体积的质量, 与沉积物的含水量、有机质含量关系密 切. 通常采用样品干重 $\left(m_{\text {干 }}\right)$ 与体积 $(V)$ 之比, 公式为:

$$
\operatorname{Dbd}=m_{\text {干 }} / V
$$

2.3.2 磁化率分析 沉积物冻干磨碎后装人 $2 \mathrm{~cm} \times 2 \mathrm{~cm} \times 2 \mathrm{~cm}$ 的样品盒中压实密封后, 进行磁性测量. 沉 积物磁化率使用英国 Bartington-MS2 磁化率仪测定沉积物样品的低频值 (频率为 $0.47 \mathrm{kHz}$ ) 和高频值 (频率 为 $4.7 \mathrm{kHz}$ ), 为提高数据的可靠性及精确性, 每个样品均测两次, 每次测两遍.

低频质量磁化率 $\chi_{\mathrm{Lf}}$ 的计算公式为: 


$$
\chi_{\mathrm{Lf}}=\chi \times 10 / M
$$

式中, $\chi_{\mathrm{Lf}}$ 单位是 $10^{-8} \mathrm{~m}^{3} / \mathrm{kg}, M$ 为样品质量.

2.3.3 粒度分析 沉积物粒度实验采用英国 MALVERN 公司生产的 Mastersiezr 2000 激光粒度仪进行测试. 实验过程如下: 取适量的沉积物湿样放置于 $100 \mathrm{ml}$ 的烧杯中 (根据样品中有机质及碳酸盐含量酶量增减); 烧杯中加人 $10 \sim 20 \mathrm{ml}$ 浓度为 $30 \%$ 的 $\mathrm{H}_{2} \mathrm{O}_{2}$, 放置于 $80^{\circ} \mathrm{C}$ 水浴锅中加热, 以去掉样品中的有机质; 往烧杯中 加人 $10 \mathrm{ml}$ 浓度为 $10 \%$ 的盐酸, 以去除碳酸盐, 持续加热到没有气泡生成; 用蒸馏水注满烧杯, 静置 $10 \mathrm{~h}$ 以 上; 抽去顶部上清液, 加人 $10 \mathrm{ml} 10 \%$ 的六偏磷酸钠 (分散剂) 溶液, 并将烧杯放人超声波发生器中水浴震 荡 $15 \mathrm{~min}$; 上机测试.

2.3.4 元素分析 将真空冷冻干燥后的样品研磨至 80 目左右的粉末状, 用锡囊封装好直接送人 EA 3000 (Euro Vector, Italy) 型元素分析仪,进行总碳 (TC) 和总氮 (TN) 的测定. 将真空冷冻干燥后样品研磨至 80 目左右的粉末状, 取适量样品放人标准银杯中, 滴加 2 滴 $5 \%$ 的 $\mathrm{HCl}$, 置于电热板上 $60^{\circ} \mathrm{C}$ 烘 $2 \mathrm{~h}$; 之后再滴加 $1 \sim 2$ 滴, 将电热板温度调至 $40^{\circ} \mathrm{C}$, 直至将样品蒸干, 后将样品用锡囊封装好送人 EA 3000 (Euro Vector, Italy) 型元素分析仪, 进行总有机碳 (TOC) 含量测定. 沉积物 TC 与 TOC 含量之差为 TIC 含量. 根据该仪器 对标准物质的重复测试结果, 相对误差在 $0.1 \%$ 以内, 表明测试结果具有较高的可信度. 该实验在中国科学 院南京地理与湖泊研究所湖泊与环境国家重点实验室内完成.

2.3.5 稳定同位素分析 有机碳稳定同位素 $\left(\delta^{13} \mathrm{C}_{\text {org }}\right)$ 分析: 取一定量的冻干研磨后的沉积物样品, 加人 $10 \%$ 的稀盐酸去除样品中碳酸盐后, 用去离子水反复清洗至中性烘干, 研磨至 80 目以下. 将样品用锡囊封装好, 经过 Flash EA1112 元素分析仪燃烧后气体送人 DeltaPlus Advantage (Thermo Finnigan MAT, Germany) 同位 素质谱仪. 无机碳稳定同位素分析: 样品的碳酸盐碳 $\left(\delta^{13} \mathrm{C}_{\text {carb }}\right)$ 、氧同位素 $\left(\delta^{18} \mathrm{O}_{\text {carb }}\right)$ 分析采用离线分析方法, 均在中国科学院南京地质古生物研究所稳定同位素实验室测试完成. 样品真空冷冻干燥后, 研磨成 100 目 左右的粉末状, 采用过饱合磷酸法,将收集到的 $\mathrm{CO}_{2}$ 气体在 Thermo-Fisher MAT 253 (Germany) 同位素质谱 仪上测定 $\delta^{13} \mathrm{C}_{\text {carb }}, \delta^{18} \mathrm{O}_{\text {carb }}$, 计算结果以 VPDB 为标准.

\section{4 数据处理与作图}

本章节所有数据均采用 Excel 2016 进行处理及汇总, 采用 SPSS 19.0 进行统计分析. 博斯腾湖区位、等 深线 ${ }^{[21]}$ 及采样点位采用 ArcGIS 10.0 \& CorelDRAW X7 软件制图, 其余图均由 Grapher 7 \& CorelDRAW X7 软件绘制.

\section{3 年代序列的建立}

人工放射性核素 ${ }^{137} \mathrm{Cs}$ 与自然放射性核素 ${ }^{210} \mathrm{~Pb}$ 已被广泛应用于百年尺度上的湖泊沉积物的定年研究, 为湖泊及其流域环境变化重建研究提供了年代学基础 ${ }^{[27-30]}$. 在很多情况下, 湖底沉积物 (尤其表层沉积物) 常常受到风浪、生物、人为清淤等干扰, 使得影响 ${ }^{210} \mathrm{~Pb}$ 沉积通量的原因很复杂, 采用单一的 CIC 模式 (constant initial concentration, 恒定沉积通量) 和 CRS 模式 (constant rate of supply, 恒定放射性通量) 计算得 到的年代容易偏离 ${ }^{137} \mathrm{Cs}$ 时标, 给定年带来困难 ${ }^{[31-32]}$. 因此, 在实际应用中多采用复合模式 ${ }^{[31,33]}$ 来解决时标 偏离问题, 即根据 ${ }^{137} \mathrm{Cs}$ 的 1963 年时标深度把沉积岩芯分为上下两段, 利用 ${ }^{210} \mathrm{~Pb}_{\mathrm{ex}}$ 活度值, 分别采用不同的 公式进行年代计算.

岩心表层至 ${ }^{137} \mathrm{Cs}$ 时标 (1963 年) 深度之间采用以下公式计算:

$$
\begin{gathered}
T_{m}=T_{0}+\lambda^{-1} \ln \left[1+\left(A_{0}-A_{m}\right) P^{-1} \cdot \lambda\right] \\
P=\frac{-\lambda\left(A_{0}-A_{w}\right)}{1-\mathrm{e}^{-\lambda\left(T_{0}-1963\right)}} \\
A_{0}=\sum_{x=1}^{n} C_{x} \cdot \rho_{x} \\
A_{w}=\sum_{x=w}^{n} C_{x} \cdot \rho_{x}
\end{gathered}
$$

而 1963 年以下各层位样品所对应年份的计算公式为: 


$$
\begin{gathered}
T_{m}=1963-\lambda^{-1} \ln \left(A_{w} / A_{m}\right) \\
A_{m}=\sum_{x=m}^{n} C_{x} \cdot \rho_{x}
\end{gathered}
$$

式中, $T_{m}$ 为 $m$ 质量深度对应的年代, $T_{0}$ 为沉积物采样年份, $\lambda$ 为 ${ }^{210} \mathrm{~Pb}$ 的衰变常数 $0.03114, A_{0}$ 为整个沉积岩 芯 ${ }^{210} \mathrm{~Pb}_{\mathrm{ex}}$ 累积量 $\left(\mathrm{Bq} / \mathrm{cm}^{2}\right), A_{m}$ 为 $m$ 质量深度以下 ${ }^{210} \mathrm{~Pb}_{\mathrm{ex}}$ 累积量 $\left(\mathrm{Bq} / \mathrm{cm}^{2}\right), C_{x}$ 为 $x$ 质量深度的 ${ }^{210} \mathrm{~Pb}_{\mathrm{ex}}$ 活度 $(\mathrm{Bq} / \mathrm{kg}), \rho_{x}$ 为 $x$ 质量深度的样品容重 $\left(\mathrm{g} / \mathrm{cm}^{2}\right), A_{w}$ 为 1963 年所对应的 $w$ 层位以下 ${ }^{210} \mathrm{~Pb}_{\mathrm{ex}}$ 累积量 $\left(\mathrm{Bq} / \mathrm{cm}^{2}\right)$.

博斯腾湖 B3、B5、B10 岩芯沉积物的 ${ }^{137} \mathrm{Cs}$ 存在多个蓄积峰 (图 2), 这与陈发虎等 ${ }^{[9]}$ 、郑柏颖等 ${ }^{[26]}$ 所测 定的结果一致, 具有较好的对比性, 说明博斯腾湖沉积物 ${ }^{137} \mathrm{Cs}$ 活度分布具有自身的特点, 跟国内外其他湖泊 所呈现的单峰态或双峰态有区别. 原因是博斯腾湖紧邻中国核试验基地,该区不仅受到全球核试验的影响, 还受当地核试验的影响. 据文献 [ 9]报道, 中国 3 个主要的核试验期分别为 1967-1970、1973 和 1976 年, 这 些时标在本研究中的 B3、B5、B10 岩芯 ${ }^{137} \mathrm{Cs}$ 曲线上均有体现.

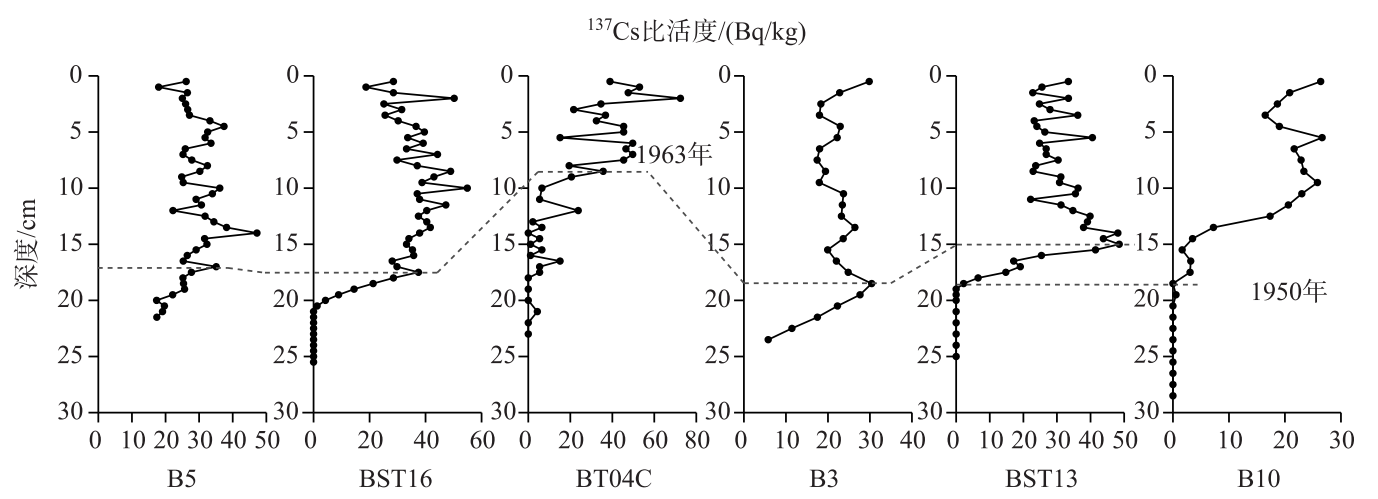

图 2 博斯腾湖不同岩芯 ${ }^{137} \mathrm{Cs}$ 测定结果

Fig. $2{ }^{137}$ Cs changing sequence of different cores in Lake Bosten

如图 3 所示, B3、 B5 和 $\mathrm{B} 10$ 钻孔中 ${ }^{210} \mathrm{~Pb}_{\mathrm{ex}}$ 的比活度随深度的增加而呈指数下降, 说明博斯腾湖具有较为 稳定的沉积环境. 经 CRS 模式的计算, 结果与已确定的 ${ }^{137} \mathrm{Cs}$ 时标有较大的偏离, 为此本研究采用了复合模
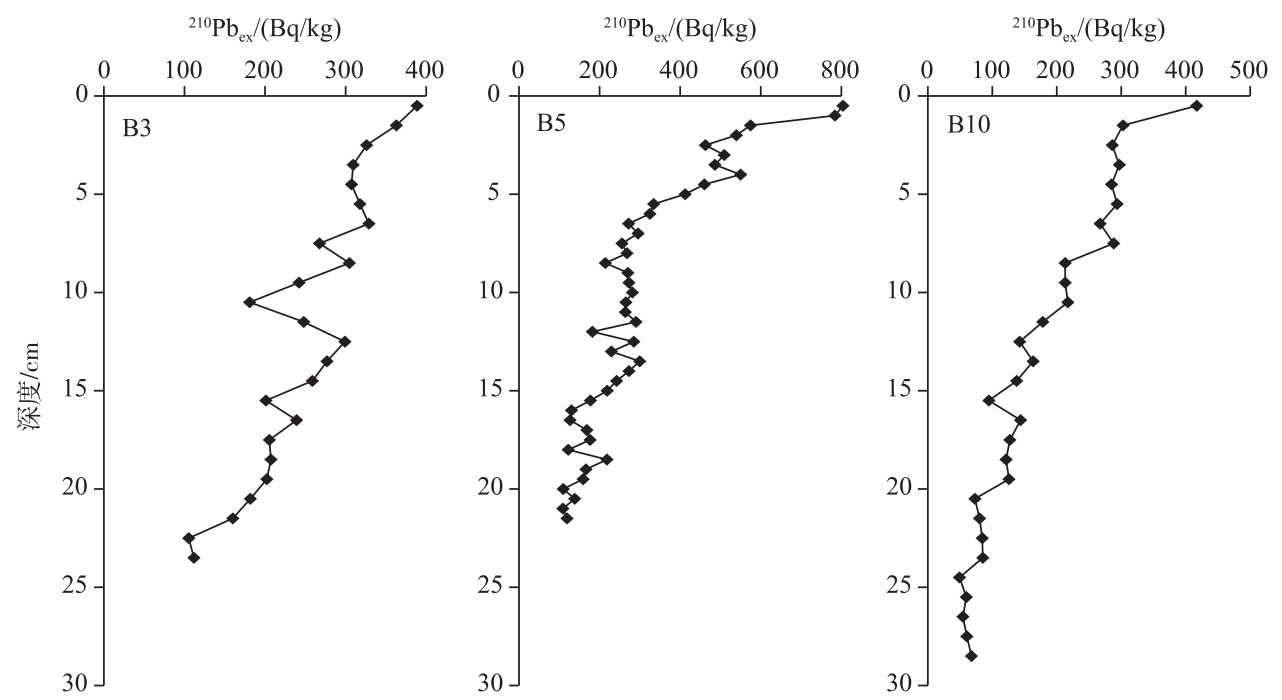

图 3 博斯腾湖 $\mathrm{B} 3 、 \mathrm{~B} 5 、 \mathrm{~B} 10$ 孔 ${ }^{210} \mathrm{~Pb}_{\mathrm{ex}}$ 活度变化

Fig. 3 Variations of ${ }^{210} \mathrm{~Pb}_{\mathrm{ex}}$ activities in the B3, B5, B10 cores from Lake Bosten 
式进行年代的确定. 对于 B10 钻孔, 由于 1963 年的 ${ }^{137}$ Cs 时标不明显, 所以选取 1950 年时标进行计算. 对于 定年结果, 我们将 $\delta^{18} \mathrm{O}_{\text {carb }}$ 数据与湖面蒸发数据进行了验证 ${ }^{[19]}$, 发现两者具有显著的正相关关系, 证实本湖 钻孔的年代序列是可靠的. 3 个孔位的复合模式年代及沉积速率 $\left(\mathrm{SAR}, \mathrm{g} /\left(\mathrm{m}^{2} \cdot \mathrm{a}\right)\right.$, 在空间上表现出了一定 的差异性 (图 4) : B3 岩芯, 1890 年以来沉积速率逐步升高; B5 岩芯, 1870-1950 年沉积速率基本处于一个 较低的平稳状态, 1950-2000 年沉积速率快速升高, 2000 年以来沉积速率不断下降; B10 岩芯, 1860-1940 年沉积速率升高较快, 1940-1960 年又出现下降, 而 1960 年以来呈现出稳步上升的趋势. 总体来看, B3、B5 和 B10 3 个钻孔近百年以来, 沉积速率表现出升高的趋势, 尤其是 1960-2000 年期间最为明显.
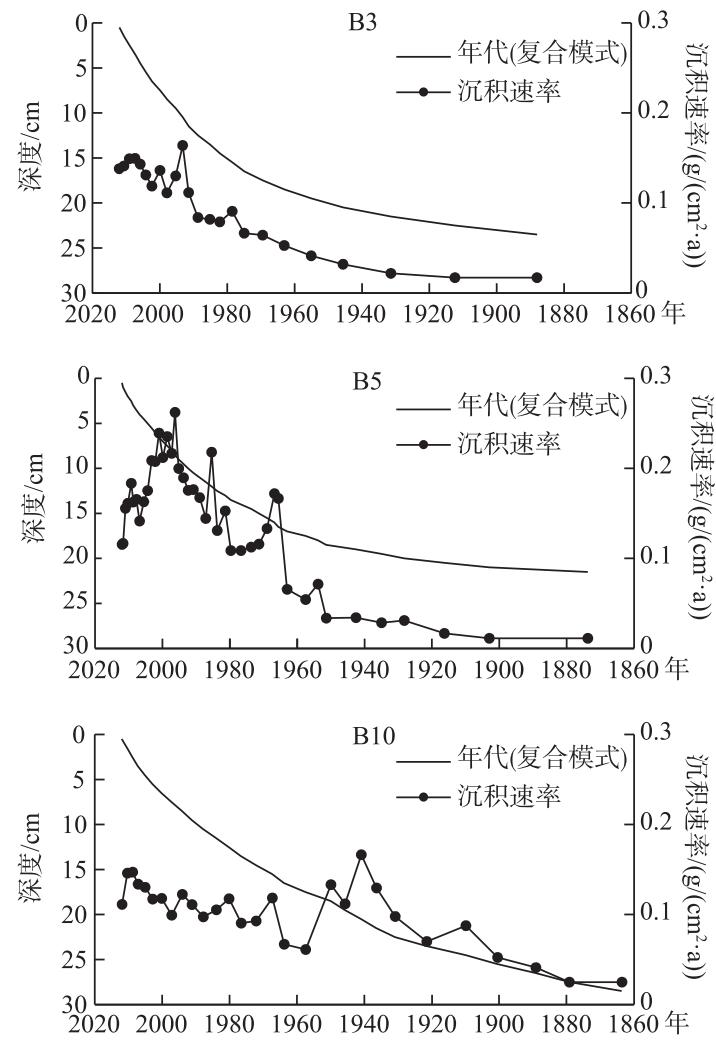

图 4 博斯腾湖 3 个采样点的年代和沉积速率

Fig.4 Age-depth plot and dry mass accumulation rate of three sediment cores in Lake Bosten

\section{0 年以来博斯腾湖碳沉积演变过程分析}

依据上述三孔各年代序列和沉积速率变化,结合沉积物各环境代用指标, 1860 年以来博斯腾湖碳沉积 变化过程可划分为以下 5 个阶段 (图 5):

阶段 I : 1860-1910 年. 该阶段博斯腾湖沉积速率较低, 沉积速率范围为 $0.01 \sim 0.09 \mathrm{~g} /\left(\mathrm{m}^{2} \cdot \mathrm{a}\right)$, 其中 B10 孔沉积速率要高于 B3、B5 孔. 磁化率、中值粒径较为稳定, TOC 含量相对较低, C/N 相对较高, 尤其是 B10 孔, 反映出此时博斯腾湖有较多陆源有机质的输人, 可能与流域初期屯田旺荒有关 ${ }^{[34]}$. B3、B5 孔 TIC 含 量变化不大, 在 $5.4 \% \sim 6.0 \%$ 之间浮动, 但是 $\mathrm{B} 10$ 孔呈现出下降的趋势, 而且 $\delta^{13} \mathrm{C}_{\text {carb }}$ 和 $\delta^{18} \mathrm{O}_{\text {carb }}$ 同步偏负, 可能 是由西北部湖区有较多径流的注人、湖面有效湿度增加导致的.

阶段 II : 1910-1950 年. 这一时期 B3、B5 孔沉积速率缓慢升高, 而 B10 孔快速升高, 平均沉积速率达 $0.12 \mathrm{~g} /\left(\mathrm{cm}^{2} \cdot \mathrm{a}\right)$, 反映出博斯腾湖西部浅水域明显高于东部深水区. B3、B5 孔磁化率波动较大, 中值粒径缓 慢下降,沉积物颗粒变细, 其他指标变化不明显; 而 B10 孔碳沉积环境变化很大, 磁化率快速升高, 中值粒径 
变大, 沉积物颗粒明显变粗, $\delta^{13} \mathrm{C}_{\text {org }}$ 明显偏正, $\mathrm{C} / \mathrm{N}$ 依然呈现高值 (11 13), 说明此时西北湖区水域有大量外 源物质输人, 而 TOC 含量明显下降, 说明此时湖泊的初级生产力较低, 内源贡献相对较小. 值得注意的是, B10 孔在此阶段出现了大量的螺类壳体 (图 $5 \mathrm{j}$ ), 这些以扁螺为主的软体动物反映出当时博斯滕湖西北水域 水深较浅、水体盐度较低. 硅藻、摇蚊等指标也反映了博斯腾湖此时处于中性淡水环境, 气候比较温暖, 而且 降水可能大于蒸发 ${ }^{[35-36]}$. B10 孔沉积物中 TIC 含量此时明显降低, $\delta^{13} \mathrm{C}_{\text {carb }}$ 和 $\delta^{18} \mathrm{O}_{\text {carb }}$ 指标也同步降低, 也说明 此时博斯腾湖较为湿润.

阶段 III : 1950-1980 年. 1950 年后, 博斯腾湖流域内人口增加, 农业活动增强, 焉耆盆地屯田怎荒面价 快速增加, 导致该湖沉积速率整体上升, 虽然 B10 孔较前一阶段有所下降. 三孔 TOC、TIC 含量均呈现升高的 趋势, 尤其是 B 10 孔升高最快; $\mathrm{C} / \mathrm{N}$ 较为一致, 且比较稳定, 反映出湖泊内源贡献在增加, 而且 $\delta^{13} \mathrm{C}_{\mathrm{org}}$ 也出现 了偏负的趋势; 同时, 磁化率和中值粒径也同步出现了下降的趋势, 表明陆源组分的输人相对前一阶段要少. 农业活动导致一些较细的颗粒带人湖泊, 同时大量的营养盐也随径流被带人湖内, 造成此时博斯腾湖初级 生产力升高 ${ }^{[37]}$, 内源有机碳贡献增加. 这一时期, 湖泊水位持续下降, 湖面较低, 加上气温的逐年升高, 造成 湖面蒸发加强, 使得湖水析出大量碳酸盐, 尤其在夏季, $\delta^{13} \mathrm{C}_{\text {carb }}$ 和 $\delta^{18} \mathrm{O}_{\text {carb }}$ 同步偏正也证实了这一点.

阶段 IV : 1980-2002 年. 此阶段三孔呈现出快速的沉积速率, 尤其湖泊东部水域最为明显. 三孔的磁化 率和中值粒径较为接近, 进一步缓慢下降. TOC 含量均呈现升高的趋势, 西部湖区要高于东部湖区, 而且 $\mathrm{C} / \mathrm{N}$ 值较低、 $\delta^{13} \mathrm{C}_{\text {org }}$ 偏负, 说明此时湖泊西部水域初级生产力要高于东部深水区, 这可能是由于东部深水区 远离河流人湖处, 营养相对贫乏, 浮游植物等生物量相对较低, 造成内源有机碳相对较少, 而外源比例较高. 夏军等 ${ }^{[38]}$ 研究指出,博斯腾湖西部水域在 $1980 \mathrm{~s}$ 末开始出现水体富营养化现象, 而东部远离河口区水质相 对较好. TIC 及其稳定同位素较为稳定, 数值均比较高, 表明湖面蒸发较强, 气候比较温暖, 这与当地的气象 资料记录一致.

阶段 $V: 2002$ 年以来. 博斯腾湖沉积速率相对较高, 其中 B 5 呈现出下降的趋势. B3、B10 孔各指标具有 很好的对应性, 而与 B5 表现出了相反的趋势, 说明最近 10 年博斯腾湖西部水域沉积环境与东部水域有较 大不同. $\mathrm{C} / \mathrm{N}$ 和 $\delta^{13} \mathrm{C}_{\text {org }}$ 数值说明该湖此时以内部生产力为主, 外源有机质贡献较小, 这可能与湖泊水体初级 固氮藻类的增加有关 ${ }^{[34]}$.

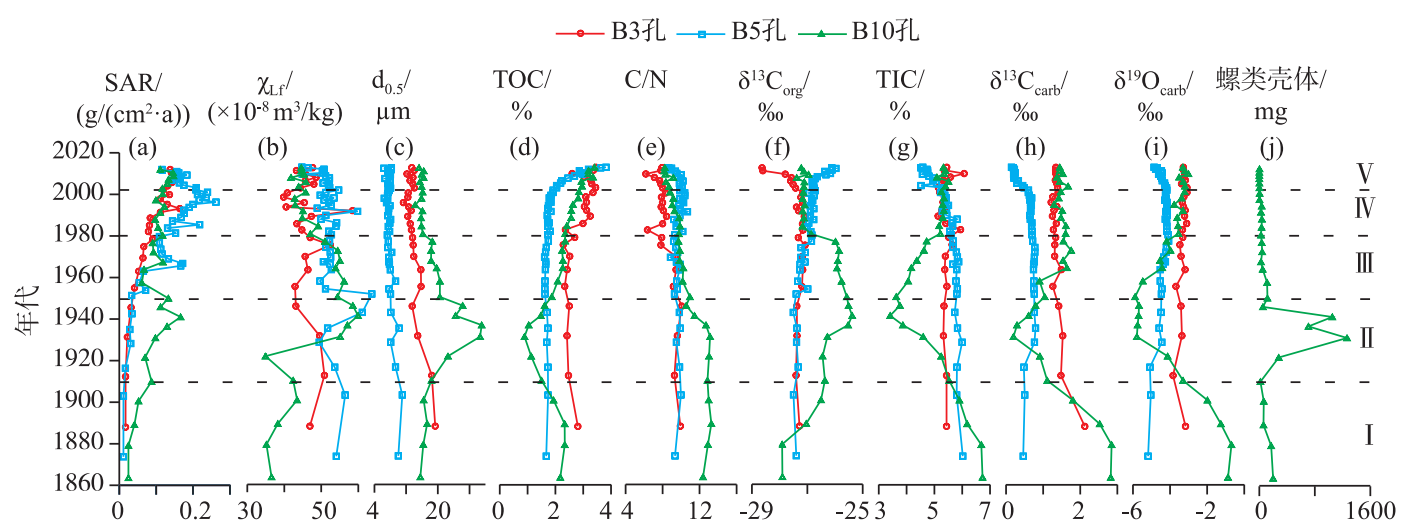

图 5 博斯腾湖 B3、B5、B10 三孔多指标综合分析

Fig.5 Analysis among multiple proxies of B3, B5 and B10 cores in Lake Bosten

\section{5 区域对比与讨论}

目前, 湖泊沉积学中有机/无机碳累积速率 (OC/IC AR) 的计算主要基于以下两种形式:1) 基于每层 有机无机碳含量 $(\mathrm{OC} / \mathrm{IC}, \%)$ 和每层的沉积物质量累积速率 ( sediment accumulation rates, SARs, $\mathrm{g} /\left(\mathrm{cm}^{2} \cdot \mathrm{a}\right)$ 相乘得到; 2 ) 线性沉积速率 ( sediment rate, SR, cm/a)、孔隙度 (porosity, $\varphi$ )、沉积物干密度 (dry sediment density $\left., \rho, g / \mathrm{cm}^{3}\right)$ 和有机 无机碳含量 $(\mathrm{OC} / \mathrm{IC}, \%)$ 相乘得到 ${ }^{[39-40]}$. 本文中 B3、B5、B10 三孔沉积物的碳沉 
积速率主要采用第 1 种形式,每孔平均 $\mathrm{OC} / \mathrm{IC}$ AR 通过下面公式获得:

$$
\overline{\mathrm{OC} / \mathrm{ICAR}}=\frac{\sum_{i=1}^{n} F_{i} \cdot \Delta t_{i} \cdot(\mathrm{OC} / \mathrm{IC} \%)_{i}}{\sum_{i=1}^{n} \Delta t_{i}}
$$

式中, $F_{i}$ 为第 $i$ 累积速率 $\left(\mathrm{g} /\left(\mathrm{cm}^{2} \cdot \mathrm{a}\right)\right), \Delta t_{i}$ 为第 $i$ 层和 $i+1$ 层的时间间隔, $(\mathrm{OC} / \mathrm{IC} \%) i$ 为第 $i$ 层的有机 无机 碳含量.

而博斯腾湖全湖平均的 $\mathrm{OC} / \mathrm{IC} \mathrm{AR}$ 取三孔的平均值来计算.

博斯腾湖沉积物中碳沉积速率整体上呈现出增加的趋势, 尤其是 1950 年以来快速增加, 意味着有更多 的碳被固定在湖泊里. 其中, B5 孔碳的累积速率要比 B3、B10 孔高, 平均值分别为 $102.0 、 80.0$ 和 $73.2 \mathrm{~g} \mathrm{C} /$ $\left(\mathrm{m}^{2} \cdot \mathrm{a}\right)$. 三孔中有机碳累积速率较为接近, 而且时间变化趋势也较为一致, 而对于无机碳累积速率, B5 孔的 平均值 $\left(73.4 \mathrm{~g} \mathrm{C} /\left(\mathrm{m}^{2} \cdot \mathrm{a}\right)\right)$ 要远大于 B3 孔 $\left(51.4 \mathrm{~g} \mathrm{C} /\left(\mathrm{m}^{2} \cdot \mathrm{a}\right)\right)$ 和 B10 孔 $\left(49.4 \mathrm{~g} \mathrm{C} /\left(\mathrm{m}^{2} \cdot \mathrm{a}\right)\right)$. B3、B5、B10 三个孔中无机碳累积速率均大于有机碳的累积速率, 并且和总碳累积速率呈现出较为一致的时间变化趋势. 总体而言, 博斯腾湖过去 150 年碳埋藏存在较大的空间差异, 东部深水区整体高于西部浅水区, 尤其是无机 碳埋藏更为突出. 因此, 在进行湖泊碳储量估算及评估工作时, 单一钻孔 (以往多于湖心深水区采样) 可能会 造成研究结果的高估判断,给相应政策的制定带来一定的误差.

早期 Stallard ${ }^{[41]}$ 估算出全球有机碳沉积速率为 $5 \mathrm{~g} \mathrm{C} /\left(\mathrm{m}^{2} \cdot \mathrm{a}\right)$, Tranvik 等 ${ }^{[42]}$ 后计算为 $10 \sim 15 \mathrm{~g} \mathrm{C} /\left(\mathrm{m}^{2}\right.$. a), 这些结果与长时间尺度的碳埋藏速率较为一致. 最近, Anderson 等 ${ }^{[43]}$ 指出早期的研究结果很可能低估 了湖泊生态系统碳的埋藏速率, 并估算出欧洲 1950 年以来所有湖泊的沉积速率约为 $60 \mathrm{~g} \mathrm{C} /\left(\mathrm{m}^{2} \cdot \mathrm{a}\right)$, 远高 于早期的计算结果. 如表 1 所示, 博斯腾湖有机碳的埋藏速率 (1950-2000 年) 为 $25.9 \mathrm{~g} \mathrm{C} /\left(\mathrm{m}^{2} \cdot \mathrm{a}\right)$, 比欧洲 湖泊和 Minnesota 中南部湖泊 (约 $50 \mathrm{~g} \mathrm{C} /\left(\mathrm{m}^{2} \cdot \mathrm{a}\right)$ ) 要低, 比欧洲农耕区湖泊(约 $100 \mathrm{~g} \mathrm{C} /\left(\mathrm{m}^{2} \cdot \mathrm{a}\right)$ ) 相差更远. 对比中国的其他湖泊, 博斯腾湖有机碳沉积速率比半干旱区湖泊 (如岱海、红碱淖、呼伦湖等 $)^{[14]}$ 要低很多 $\left(50 \mathrm{~g} \mathrm{C} /\left(\mathrm{m}^{2} \cdot \mathrm{a}\right)\right.$ ), 但是比长江中下游的大部分浅水湖 ${ }^{[4]}$ 要高 (约 $15 \mathrm{~g} \mathrm{C} /\left(\mathrm{m}^{2} \cdot \mathrm{a}\right)$ ). 造成这种空间分布格局 的原因主要跟流域气候、植被覆盖、湖泊特征 (水深、面积、地形、水化学) 及人类活动强弱 (土地利用变化、灌 溉、污水排放) 的差异有关 ${ }^{[45-47]}$.

表 1 不同时期博斯腾湖沉积物碳累积速率 $\left(\mathrm{g} \mathrm{C} /\left(\mathrm{m}^{2} \cdot \mathrm{a}\right)\right)$ 比较

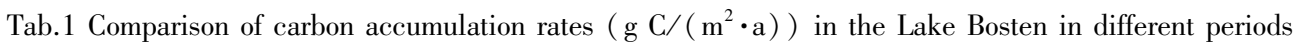

\begin{tabular}{|c|c|c|c|c|c|c|c|c|c|c|c|c|}
\hline \multirow{2}{*}{ 时期 } & \multicolumn{3}{|c|}{ B3 } & \multicolumn{3}{|c|}{ B5 } & \multicolumn{3}{|c|}{ B10 } & \multicolumn{3}{|c|}{ BST 平均值 } \\
\hline & $\mathrm{TC}$ & $\mathrm{OC}$ & IC & $\mathrm{TC}$ & OC & IC & $\mathrm{TC}$ & OC & IC & $\mathrm{TC}$ & OC & IC \\
\hline $2000-2012$ 年 & 120.2 & 44.4 & 75.9 & 125.9 & 43.8 & 82.2 & 109.2 & 40.4 & 68.8 & 118.5 & 42.8 & 75.6 \\
\hline $1950-2000$ 年 & 77.6 & 27.2 & 50.3 & 110.3 & 26.5 & 83.8 & 72.6 & 24.0 & 48.6 & 86.8 & 25.9 & 60.9 \\
\hline $1860-1950$ 年 & 17.3 & 5.6 & 11.8 & 16.7 & 3.8 & 13.0 & 50.8 & 12.9 & 37.9 & 28.3 & 7.4 & 20.9 \\
\hline
\end{tabular}

20 世纪以来, 全球很多湖泊沉积物中出现有机碳埋藏快速增加的现象 ${ }^{[43-44,48]}$, 这可能主要与温度的升 高、降水的增多及人类活动的增强等有关 ${ }^{[46-47,49]}$.首先, 温度变化对于湖泊碳埋藏的影响一般从两个方面起 作用: 一是温度升高促进浮游生物的生长, 生长季延长, 提高了湖泊初级生产力, 加上随温度升高从流域中 带人很多外源性有机碳, 导致更多的有机碳埋藏在沉积物中 ${ }^{[44,49]}$; 二是温度升高会导致有机质的矿化, 加速 沉积物中有机碳的分解, 从而减少有机碳的埋藏 ${ }^{[50]}$. 统计分析显示, 博斯腾湖沉积物有机碳沉积速率与温 度具有极显著相关性 ${ }^{[19]}$, 这说明流域气温的升高, 促进了沉积物中有机碳的埋藏. 整体上, 高纬度地区和低 纬度地区湖泊沉积物有机碳埋藏与温度呈负相关, 有机碳的埋藏效率较低 ${ }^{[51-52]}$; 而中纬度湖区两者则主要 呈现正相关关系, 尤其是区域尺度范围上温度升高促进了沉积物有机碳的埋藏 ${ }^{[53-54]}$.

一般来讲, 降水量增加会提高流域内土壤和植被的碳储量, 随地表径流进人湖泊的有机碳将增加, 这一 现象在国内外很多湖泊中已被证实 ${ }^{[47,49]}$. 然而, 通过分析博斯腾湖沉积物有机碳与降水量的关系, 发现两者 相关性并不显著 ${ }^{[19]}$, 这主要与博斯腾湖地处西北干旱区, 年均降水量较少, 大气降水对该湖及流域影响相对 
较小,加之人类在该湖上下游修建的各种水利调控设施干扰了自然降水对湖泊的影响有关. 对于以外源性 为主的湖泊来讲, 降水量的增加无疑会提高湖泊沉积物有机碳的埋藏, 而对于内源性湖泊而言, 降水量的影 响可能就没有那么直接.

此外, 多数学者认为近现代湖泊沉积物中有机碳的快速增加是气候变暖和人类干扰共同作用导致的, 但 Anderson 等 $^{[43]}$ 近期研究指出强烈的流域农业活动是其变化的主要驱动力, 而非气候因素. 本文研究发 现, 博斯腾湖沉积物有机碳的埋藏速率与其质量沉积速率高度相关, 说明流域内农业活动及相关土地利用 方式对它们有显著影响, 反映出该湖在过去的 100 年里初级生产力的变化深受湖周人类活动的影响 ${ }^{[1,26]}$. 总体而言, 我国各大湖区近百年 (尤其是 1950 年以来) 湖泊沉积物有机碳的增加得益于气候变化和人类活 动的双重作用 ${ }^{[4,47,49,55]}$.

博斯腾湖近百年来沉积物无机碳的累积速率明显高于有机碳累积速率, 1950 年以前和 1950-2000 年 数值高达 20.9 和 $60.9 \mathrm{~g} \mathrm{C} /\left(\mathrm{m}^{2} \cdot \mathrm{a}\right)$. 这比新疆地区其他湖泊, 如艾比湖 $\left(13 \mathrm{~g} \mathrm{C} /\left(\mathrm{m}^{2} \cdot \mathrm{a}\right)\right)$ 、巴里坤湖 $(11 \mathrm{~g} \mathrm{C} /$ $\left.\left(\mathrm{m}^{2} \cdot \mathrm{a}\right)\right)$ 、巴伦拓海 $\left(15 \mathrm{~g} \mathrm{C} /\left(\mathrm{m}^{2} \cdot \mathrm{a}\right)\right)$ 沉积速率要快很多, 然而却低于半干旱区的岱海 $\left(124 \mathrm{~g} \mathrm{C} /\left(\mathrm{m}^{2} \cdot \mathrm{a}\right)\right)$ 和 红碱淖 $\left(111 \mathrm{~g} \mathrm{C} /\left(\mathrm{m}^{2} \cdot \mathrm{a}\right)\right)^{[14]}$; 而在我国东部平原湿润地区, 多为淡水湖泊, 大部分湖泊中碳酸盐的含量很 低, 甚至湖水达不到碳酸盐析出结晶的条件 ${ }^{[44-45]}$. 近期研究发现, 1950 年以来, 博斯腾湖沉积物无机碳的埋 藏主要与流域温度、湖水初级生产力及焉耆盆地水土大开发有关, 尤其是上游开都河流域的土壤洗盐过程 对博斯腾湖碳酸盐的析出具有重要影响 ${ }^{[19]}$. 目前, 对于我国各大湖区湖泊沉积物无机碳的研究相对较少, 函待加强区域空间及时间差异的对比分析,并深层次探讨其分布格局的影响因素.

现将博斯腾湖有机碳和无机碳全部考虑在内, 那么该湖沉积物碳埋藏速率分别为 $28.3 \mathrm{~g} \mathrm{C} /\left(\mathrm{m}^{2} \cdot \mathrm{a}\right)$

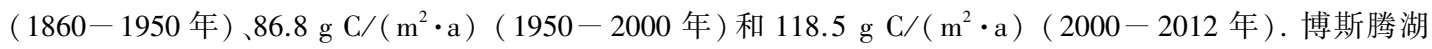
1860 年以来沉积物平均碳累积速率为 $85 \mathrm{~g} \mathrm{C} /\left(\mathrm{m}^{2} \cdot \mathrm{a}\right)$, 结合湖泊总面积 $1005 \mathrm{~km}^{2}$ (采样时湖泊面积), 可以 估算出 1860 年以来该湖共埋藏无机碳 $8.9 \mathrm{Tg}$ C、有机碳 $4.1 \mathrm{Tg}$ C、总碳 $13.0 \mathrm{Tg}$ C. 由此可知,干旱区湖泊沉积 物中存储着大量的碳,无机碳含量远高于有机碳含量,在评价和估算陆地碳储量中具有重要影响.

\section{6 参考文献}

[ 1 ] Wang SM, Zhang ZK. Recent advances in lake sedimentation and environmental evolution in China. Chinese Science Bulletin, 1999, 44(6) : 579-587. [王苏民, 张振克. 中国湖泊沉积与环境演变研究的新进展. 科学通报, 1999, 44 (6) : 579-587.]

[ 2 ] Shen J, Xue B, Wu JL et al eds. Lake sedimentation and environmental evolution. Beijing: Science Press, 2010. [沈吉, 薛斌, 吴敬禄等. 湖泊沉积与环境演化. 北京: 科学出版社, 2010.]

[ 3 ] Chen JA, Wan GJ, Wang FS et al. Study on environmental records in modern lacustrine sediments. Science in China: Series D, 2002, 32(1): 73-80. [ 陈敬安, 万国江, 汪福顺等. 湖泊现代沉积物碳环境记录研究. 中国科学: D 辑: 地 球科学, 2002, 32(1): 73-80.]

[ 4 ] Dean W. The carbon cycle and biogeochemical dynamics in lake sediments. Journal of Paleolimnology, 1999, 21 (4): 375-393.

[ 5 ] Meyers PA. Applications of organic geochemistry to paleolimnological reconstructions: a summary of examples from the Laurentian Great Lakes. Organic Geochemistry, 2003, 34(2) : 261-289.

[ 6 ] Zhu C, Pan JM, Lu B et al. Source indication and accumulative effect of sedimentary organic matter in the Changjiang Estuary, the old Huanghe River subaqueous delta and the East China Sea shelf. Journal of Marine Sciences, 2005, 23(3): 36-46. [ 朱纯, 潘建明, 卢冰等. 长江、老黄河口及东海陆架沉积有机质物源指标及有机碳的沉积环境. 海洋学研 究, 2005, 23(3) : 36-46.]

[ 7 ] Yu PS, Xue B, Pan JM et al. Impact of sediment grain size on the distribution of organic matter in Changjiang River Estuary. Journal of Marine Sciences, 2011, 29(3): 202-208. [于培松, 薛斌, 潘建明等. 长江口和东海海域沉积物粒径 对有机质分布的影响. 海洋学研究, 2011, 29(3): 202-208.]

[ 8 ] Chen FH, Huang XZ, Yang ML et al. Westerly dominated Holocene climate model in arid central Asia—Case study on Bosten Lake, Xinjiang, China. Quaternary Sciences, 2006, 26(6): 881-887. [ 陈发虎, 黄小忠, 杨美临等. 亚洲中部干 旱区全新世气候变化的西风模式一以新疆博斯腾湖记录为例. 第四纪研究, 2006, 26(6): 881-887.] 
[ 9 ] Chen FH, Huang XZ, Zhang JW et al. Humid Little Ice Age in arid central Asia documented by Bosten Lake, Xinjiang. Science in China: Series D, 2007, 37(1): 77-85. [陈发虎, 黄小忠, 张家武等. 新疆博斯腾湖记录的亚洲内陆干旱 区小冰期湿润气候研究. 中国科学: D 辑: 地球科学, 2007, 37(1): 77-85.]

[10] Ren YQ, Wang CH, Li RB et al. Ecological environment change recorded by sediment n-alkane and $\delta^{13} \mathrm{C}_{\text {org }}$ of Lake Bosten since late of early Holocene. Quaternary Sciences, 2014, 34(2): 425-433. [任雅琴, 王彩红, 李瑞博等. 有机质饱 和烃和 $\delta^{13} \mathrm{C}_{\text {org }}$ 记录的博斯腾湖早全新世晚期以来生态环境演变. 第四纪研究, 2014, 34(2): 425-433.]

[11] Zhang CJ, Zheng MP, Prokopenko A et al. The palaeoenvironmental variation from the high-resolution record of the holocene sediment carbonate and isotopic composition in Bosten Lake and responding to glacial activity. Acta Geologica Sinica, 2007, 81(12) : 1658-1671. [张成君, 郑绵平, Prokopenko A 等. 博斯腾湖碳酸盐和同位素组成的全新世古环境演 变高分辨记录及与冰川活动的响应. 地质学报, 2007, 81(12) : 1658-1671.]

[12] Zhang JW, Wang JL, Guo XY et al. Paleoclimatic significance of oxygen isotope composition of carbonates from sediment core at Bosten Lake, Xinijang, China. Quaternary Sciences, 2010, 30(6) : 1078-1087. [张家武, 王君兰, 郭小燕等. 博斯腾湖全新世岩芯沉积物碳酸盐氧同位素气候意义. 第四纪研究, 2010, 30(6) : 1078-1087.]

[13] Zhong W, Zhang J, Shu Q. Palaeoclimatic Oscillation since about 12000 yr B.P. at Bosten Lake, Southern Xinjiang, NW China, reflected in abundance and stable isotope composition of sediment carbonate. Journal of Xinjiang University, 2000, 17 (1) : 72-80. [ 钟巍, 张进, 舒强. 近 12000aB.P. 以来博斯腾湖地区古气候变化的沉积物稳定同位素及其他指标 的记录(英文). 新疆大学学报: 自然科学版, 2000, 17(1): 72-80.]

[14] Lan JH. Carbon burials in lake sediments over the arid-semiarid region, Northwestern China [Dissertation]. Beijing: University of Chinese Academy of Sciences, 2013. [蓝江湖. 我国西北干旱-半干旱区湖泊碳埋藏研究 [学位论文]. 北 京: 中国科学院大学, 2013.]

[15] Yu ZT, Wang XJ, Zhao CY et al. Source characterization of organic carbon using elemental, isotopic and n-alkanes proxies in surface sediment from Lake Bosten, Xinjiang. J Lake Sci, 2015, 27( 5) : 983-990. DOI:10.18307/2015.0526. [于志 同, 王秀君, 赵成义等. 基于多指标分析的博斯腾湖表层沉积物有机碳来源. 湖泊科学, 2015, 27(5): 983-990.]

[16] Yu ZT, Wang XJ, Zhang EL et al. Spatial distribution and sources of organic carbon in the surface sediment of Bosten Lake, China. Biogeosciences, 2015, 12(22): 6605-6615.

[17] Yu ZT, Wang XJ, Zhao CY et al. Spatial variations of inorganic carbon and isotopes in the surface sediment of the Lake Bosten. J Lake Sci, 2015, 27(2) : 250-257. DOI:10.18307/2015.0208. [于志同, 王秀君, 赵成义等. 博斯腾湖表层 沉积物无机碳及其稳定同位素空间异质性. 湖泊科学, 2015, 27(2) : 250-257.]

[18] Wang XJ, Fang CL, Yu ZT et al. Seasonal variations and sources of particulate and dissolved organic carbon in Lake Bosten, Xinjiang Province. J Lake Sci, 2014, 26(4) : 552-558. DOI : 10.18307/2014.0409. [王秀君, 房传苓, 于志同等. 新疆博斯腾湖水体颗粒和溶解有机碳的季节变化及其来源初探. 湖泊科学, 2014, 26(4) : 552-558.]

[19] Yu ZT, Wang XJ, Zhao CY et al. Carbon burial in Bosten Lake over the past century: Impacts of climate change and human activity. Chemical Geology, 2015, 419: 132-141.

[20] Yu ZT, Wang XJ. Temporal variability of carbon burial and the underlying mechanisms in Bosten Lake since 1950// Wang XJ ed. Carbon cycle in the changing arid land of China. Springer Singapore, 2018: 117-132.

[21] Wu JL, Ma L, Zeng HA. Water quality and quantity characteristics and its evolution in Lake Bosten, Xinjiang over the past 50 years. Scientia Geographica Sinica, 2013, 33(2): 231-237. [吴敬禄, 马龙, 曾海鳌. 新疆博斯腾湖水质水量 及其演化特征分析. 地理科学, 2013, 33(2): 231-237.]

[22] Guo M, Wu W, Zhou X et al. Investigation of the dramatic changes in lake level of the Bosten Lake in northwestern China. Theoretical and Applied Climatology, 2015, 119: 341-351.

[23] Gao HZ, Yao YF. Quantitative effect of human activities on water level change of Bosten Lake in recent 50 years. Scientia Geographica Sinica, 2005, 25(3): 3305-3309. [ 高华中, 姚亦锋. 近 50 年来人类活动对博斯腾湖水位影响的量化 研究. 地理科学, 2005, 25(3): 3305-3309.]

[24] Wu JL, Ma L, Zeng HA. Recent changes of selected lake water resources in arid Xinjiang, Northwestern China. Quaternary Sciences, 2012, 32(1): 142-150. [ 吴敬禄, 曾海鳌, 马龙等. 新疆主要湖泊水资源及近期变化分析. 第四纪研 究, 2012, 32(1): 142-150.]

[25] Ilnur G, Hamid Y, Mamattursun E et al. The driving forces of the Bosten Lake water level variations in 1958-2012. Journal of Desert Research, 2015, 35(1) : 240-247. [伊丽努尔·阿力甫江, 海米提·依米提, 麦麦提吐尔逊·艾则孜等. 
1958-2012 年博斯腾湖水位变化驱动力. 中国沙漠, 2015, 35(1) : 240-247.]

[26] Zheng BY, Zhang EL, Gao G. The change of primary productivity of Lake Bosten in Xinjiang over the past 100 a. J Lake $S c i, 2012,24$ (3) : 466-473. DOI : 10.18307/2012.0320. [郑柏颢, 张恩楼, 高光. 近百年来新疆博斯腾湖初级生产 力的变化. 湖泊科学, $2012,24(3): 466-473$.

[27] Choudhary P, Routh J, Chakrapani G. A 100-year record of changes in organic matter characteristics and productivity in Lake Bhimtal in the Kumaon Himalaya, NW India. Journal of Paleolimnology, 2013, 49(2) : 129-143.

[28] Zeng L, Wu FC, Wan GJ et al. The distribution characteristic and environmental significance of Cesium-137 deposit profile in Chinese lacustrine sediment. J Lake Sci, 2009, 21(1) : 1-9. DOI:10.18307/2009.0101. [ 曾理, 吴丰昌, 万国江等. 中国地区湖泊沉积物中 ${ }^{137} \mathrm{Cs}$ 分布特征和环境意义. 湖泊科学, 2009, 21(1) : 1-9.]

[29] Wan GJ, Chen JA, Xu SQ et al. Indication of sudden increase of ${ }^{210} \mathrm{~Pb}_{\mathrm{ex}}$ flux on lake production: An example from Lake Chenghai, China. Science in China: Ser D: Earth Sciences, 2004, 34(2): 154-162. [ 万国江, 陈敬安, 胥思勤等. ${ }^{210} \mathrm{~Pb}_{\mathrm{ex}}$ 沉积通量突发增大对湖泊生产力的指示——以程海为例. 中国科学: D 辑: 地球科学, 2004, 34(2): 154-162.]

[30] Wan GJ, Chen JA, Wu FC et al. Coupling between ${ }^{210} \mathrm{~Pb}_{\mathrm{ex}}$ and organic matter in sediments of a nutrient-enriched lake: An example from Lake Chenghai, China. Chemical Geology, 2005, 224(4) : 223-236.

[31] Appleby PG. Chronostratigraphic techniques in recent sediments. In: Last W, Smol J eds. Tracking environmental change using lake sediments. Dordrecht: Springer, 2001: 171-203.

[32] Wan GJ, Appleby PG. Progress on fallout radiouclides as tracers in environ-ecological systems. Advance in Earth Science, 2000, 15(2) : 172-177. [万国江, Appleby PG. 环境生态系统散落核素示踪研究新进展. 地球科学进展, 2000, 15 (2) : 172-177.]

[ 33] Liu EF, Xue B, Yang XD et al. ${ }^{137} \mathrm{Cs}$ and ${ }^{210} \mathrm{~Pb}$ chronology for modern lake sediment: A case study of Chaohu Lake and Taibai Lake. Marine Geology and Quaternary Geology, 2009, 29(6): 89-94. [刘恩峰, 薛滨, 羊向东等. 基于 ${ }^{210} \mathrm{~Pb}$ 与 ${ }^{137} \mathrm{Cs}$ 分布的近代沉积物定年方法——以巢湖、太白湖为例. 海洋地质与第四纪地质, 2009, 29(6): 89-94.]

[34] Zheng BY, Cao YM, Zhang EL et al. C, N stable isotope records of environmental changes in Bosten Lake during the past 200 years. Marine Geology and Quaternary Geology, 2012, 32(6) : 165-171. [郑柏颖, 曹艳敏, 张恩楼等. 博斯腾湖 近 200 年来湖泊环境变化的有机碳氮稳定同位素记录. 海洋地质与第四纪地质, 2012, 32(6): 165-171.]

[35] Zhang EL, Zheng BY, Cao YM et al. The effects of environmental changes on chironomid fauna during the last century in Bosten Lake, Xinjiang, NW China. Fundamental and Applied Limnology / Archiv für Hydrobiologie, 2012, 180(4): 299-307.

[36] Gao G, Tang XM, Sai B eds. Evolution of ecological environment in Bosten Lake. Beijing: Science Press, 2013. [高光, 汤祥明, 赛.巴雅尔图. 博斯腾湖生态环境演化. 北京: 科学出版社, 2013.]

[37] Zheng BY, Zhang EL, Gao G. The change of primary productivity of Lake Bosten in Xinjiang over the past 100 a. J Lake $S c i, 2012,24(3): 466-473$. DOI : 10.18307/2012.0320. [ 郑柏颖, 张恩楼, 高光. 近百年来新疆博斯腾湖初级生产 力的变化. 湖泊科学, $2012,24(3): 466-473$.]

[38] Xia J, Zuo QT, Shao MC eds. Sustainable use of water resources in Bosten Lake: Theory, method, practice. Beijing: Science Press, 2013. [夏军, 左其亭, 邵民诚. 博斯腾湖水资源可持续利用: 理论. 方法. 实践. 北京: 科学出版 社, 2013.]

[39] Alin SR, Johnson TC. Carbon cycling in large lakes of the world: A synthesis of production, burial, and lake-atmosphere exchange estimates. Global Biogeochemical Cycles, 2007, 21(3) : GB3002. DOI: 10.1029/2006GB002881.

[40 ] Müller B, Maerki M, Schmid M et al. Internal carbon and nutrient cycling in Lake Baikal: sedimentation, upwelling, and early diagenesis. Global and Planetary Change, 2005, 46(1) : 101-124.

[41] Stallard RF. Terrestrial sedimentation and the carbon cycle: Coupling weathering and erosion to carbon burial.Global Biogeochemical Cycles, 1998, 12(2) : 231-257.

[42] Tranvik LJ, Dowing JA, Cotner JB et al. Lakes and reservoirs as regulators of carbon cycling and climate. Limnology and Oceanography, 2009, 54(6): 2298-2314.

[43] Anderson NJ, Dietz RD, Engstrom DR. Land-use change, not climate, controls organic carbon burial in lakes. Proceedings of the Royal Society B: Biological Sciences, 2013, 280: 20131278. DOI:10.1098/rspb.2013.1278.

[44] Dong X, Anderson NJ, Yang X et al. Carbon burial by shallow lakes on the Yangtze floodplain and its relevance to regional 
carbon sequestration. Global Change Biology, 2012, 18(7) : 2205-2217.

[45] Gui ZF. The Carbon burial of lake sediments in eastern China over the past 100 years [Dissertation]. Nanjing: Nanjing Institute of Geography \& Limnology, Chinese Academy of Sciences, 2012. [桂智凡. 近百年来中国东部地区湖泊沉积物 碳埋藏研究 [ 学位论文]. 南京: 中国科学院南京地理与湖泊研究所, 2012.]

[46] Dietz RD, Engstrom DR, Anderson NJ. Patterns and drivers of change in organic carbon burial across a diverse landscape: Insights from 116 Minnesota lakes. Global Biogeochemical Cycles, 2015, 29(5): 708-727.

[47] Zhang FJ, Yao SC, Xue B et al. Organic carbon burial in Chinese lakes over the past 150 years. Quaternary International, 2017, 438: 94-103.

[48] Heathcote AJ, Downing JA. Impacts of eutrophication on carbon burial in freshwater lakes in an intensively agricultural landscape. Ecosystems, 2012, 15(1): 60-70.

[49] Wang M, Wu JH, Chen H et al. Temporal-spatial pattern of organic carbon sequestration by Chinese lakes since 1850. Limnology \& Oceanography, 2018, 63(3) : 1283-1297.

[50] Gudasz C, Bastviken D, Steger K et al. Temperature-controlled organic carbon mineralization in lake sediments. Nature, 2010, 466(7305) : 478-481.

[51] Sobek S, Anderson NJ, Bernasconi SM et al. Low organic carbon burial efficiency in arctic lake sediments. Journal of Geophysical Research Biogeosciences, 2014, 119(6) : 1231-1243.

[52] Marotta H, Pinho L, Gudasz C et al. Greenhouse gas production in low-latitude lake sediments responds strongly to warming. Nature Climate Change, 2014, 4(6) : 467-470.

[53] Wang M, Chen H, Yu Z et al. Carbon accumulation and sequestration of lakes in China during the Holocene. Global Change Biology, 2015, 21(12) : 4436-48.

[54] Heathcote AJ, Anderson NJ, Prairie YT et al. Large increases in carbon burial in northern lakes during the Anthropocene. Nature Communications, 2015, 6: 10016.

[55] Gui ZF, Xue B, Yao SC et al. Organic carbon burial in lake sediments in the middle and lower reaches of the Yangtze River Basin, China. Hydrobiologia, 2013, 710(1): 143-156. 